\title{
Patients and practice guidelines: Keeping eyes on the horizon
}

\author{
John K Marshall MD MSc FRCPC AGAF
}

$\mathrm{T}$ he Canadian Association of Gastroenterology (CAG) recently published consensus guidelines on the management of nonhospitalized patients with active ulcerative colitis (1). Clinical practice guidelines provide useful reference standards for clinicians managing complex medical conditions. The process for developing guidelines typically convenes content experts to synthesize and evaluate available evidence, consider alternate approaches to care and generate a series of recommendations for therapy (2). Both the strength of each recommendation and the quality of evidence in its support are rated and reported in the final consensus document.

In the current issue of the Journal, Steinhart and Fernandes (3) (pages 294-296) provide a novel and invaluable patient perspective on the CAG ulcerative colitis guidelines. Importantly, patients recommended development of lay language versions of the guidelines to help inform their discussions with health care providers. The patient panel also recommended greater emphasis on quality of life as an outcome of the appraisal of evidence for and against various therapeutic approaches. Crohn's and Colitis Canada is to be commended for convening this panel post hoc, but should patients not be involved earlier in the guideline process?

There exists a rich body of literature on patient involvement in all aspects of health care decision making; however, the practical aspects of patient involvement in clinical practice guidelines remain poorly defined. Diaz del Campo et al (4) have proposed a strategy for developing patient-oriented clinical practice guidelines; however, engaging patients in the actual development of guidelines aimed at health care providers is a different concern. Kelson et al (5) have emphasized that consumer involvement in clinical practice guidelines could occur at two levels. First, the literature review that informs guideline content can specifically search for studies that consider and assess consumer and public values. Second, consumers themselves can participate formally at each stage of guideline development. The latter is more challenging because the appraisal of scientific literature and discussion of complex clinical care pathways may not be accessible to members of the public.

To be sure, both patients and content experts will be apprehensive about collaboration on guideline development. Patients may be intimidated by the scientific literature and technical discussion; however, experts may worry that important immediate clinical considerations get sidelined. Legare et al (6) reviewed the literature regarding patient participation in clinical practice guideline development and proposed a number of potential facilitators for that process. These include careful selection of participants to represent key constituencies, appropriate training of patient representatives on technical aspects of guidelines, careful selection of a chair who supports and engages patient contributions, and collaboration with relevant public agencies and organizations.

Ultimately, clinical practice guidelines strive to optimize patient outcomes by encouraging care that makes the best use of available diagnostic technology, medical and surgical therapy and knowledge of natural history. But what are optimal outcomes? Steinhart and Fernandes (3) emphasize the importance of quality of life as a broad and summative measure of benefit. This is a valid point. The benefits of many interventions are assessed in the short term by more immediate clinical metrics such as activity indexes and event rates. Yet, clinicians would do well to keep an eye on the horizon, remembering what long-term goals of care will matter most to patients.

It is arguable that the best health care decisions are made when patients and providers engage in an informed and balanced discussion of treatment alternatives, adapting available evidence and guidance to patient values and preferences. Steinhart and Fernandes, with Crohn's and Colitis Canada, should be commended for bringing this issue forward. The CAG and other Canadian national organizations guidelines may consider more formal public engagement in clinical practice guideline initiatives going forward.

\section{REFERENCES}

1. Bressler B, Marshall JK, Bernstein CN et al. Clinical practice guidelines for the medical management of non-hospitalized ulcerative colitis: The Toronto Consensus. Gastroenterology 2015;148:1035-58.

2. Singh H, Leontiadis GI, Hookey L et al. Canadian Association of Gastroenterology policy on the application for, and implementation of, clinical practice guidelines. Can J Gastroenterol Heptaol 2014;28:473-80.

3. Steinhart AH, Fernandes A. Clinical practice guidelines for the medical management of non-hospitalized ulcerative colitis: the patient perspective. Can J Gastroenterol Hepatol 2015:29:294-6.

4. Diaz del Campo, Gracia J, Biasco JA, Andradas E. A strategy for patient involvement in clinical practice guidelines: Methodological approaches. BMJ Qual Saf 2011;20:779-84.

5. Kelson M, Bastian H, Cluzeau F, et al. Integrating values and consumer involvement in guidelines with the patient at the center: Article 8 in integrating and coordinating efforts in COPD guideline development. An official ATS/ERS workshop report.

Proc Am Thorac Soc 2012;9:262-8.

6. Legare F, Boivin A, Van der Weijden T. Patient and public involvement in clinical practice guidelines: A knowledge synthesis of existing program. Med Decis Making 2011;E45-57. 


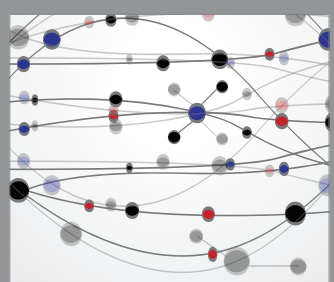

The Scientific World Journal
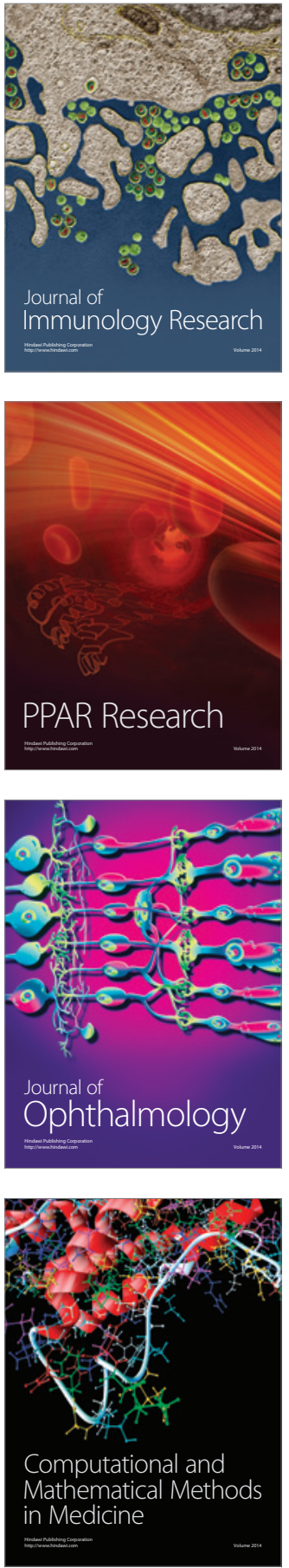

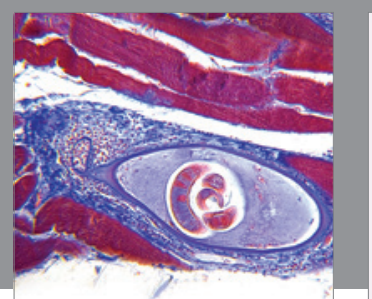

Gastroenterology Research and Practice

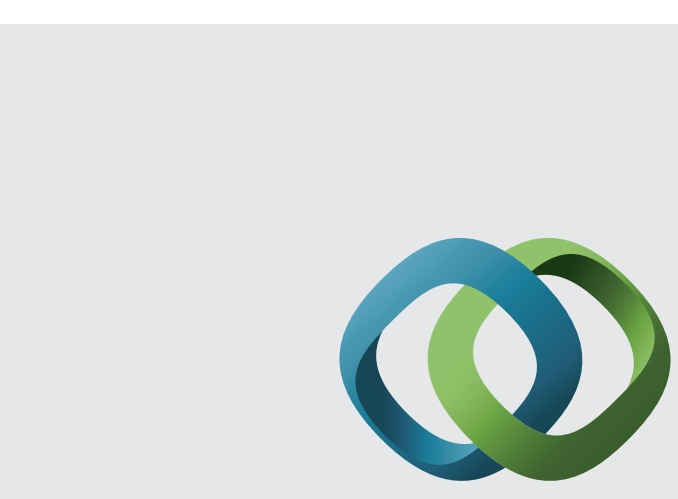

\section{Hindawi}

Submit your manuscripts at

http://www.hindawi.com
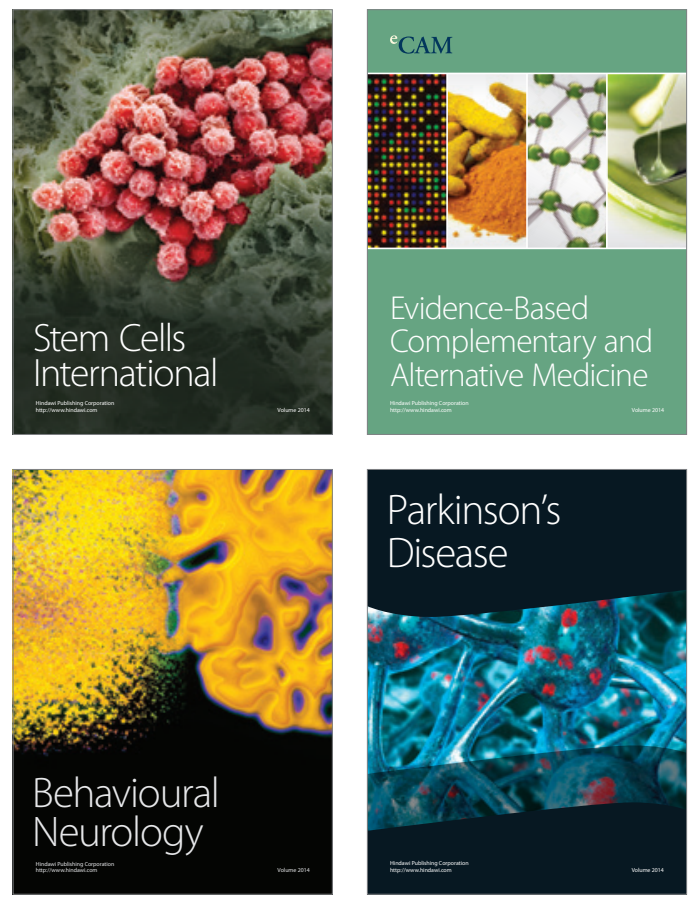
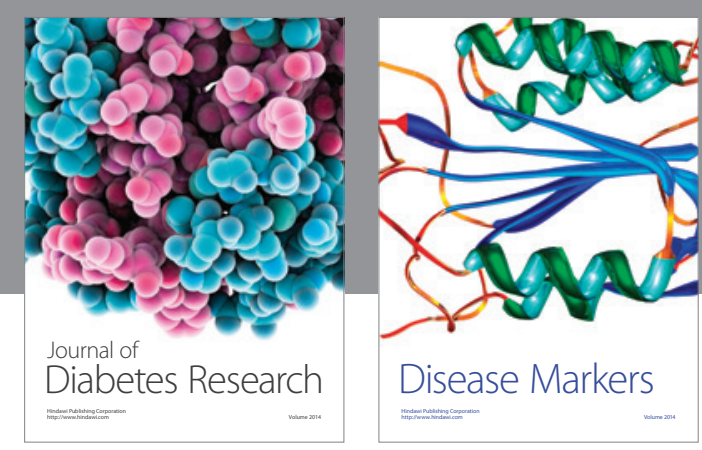

Disease Markers
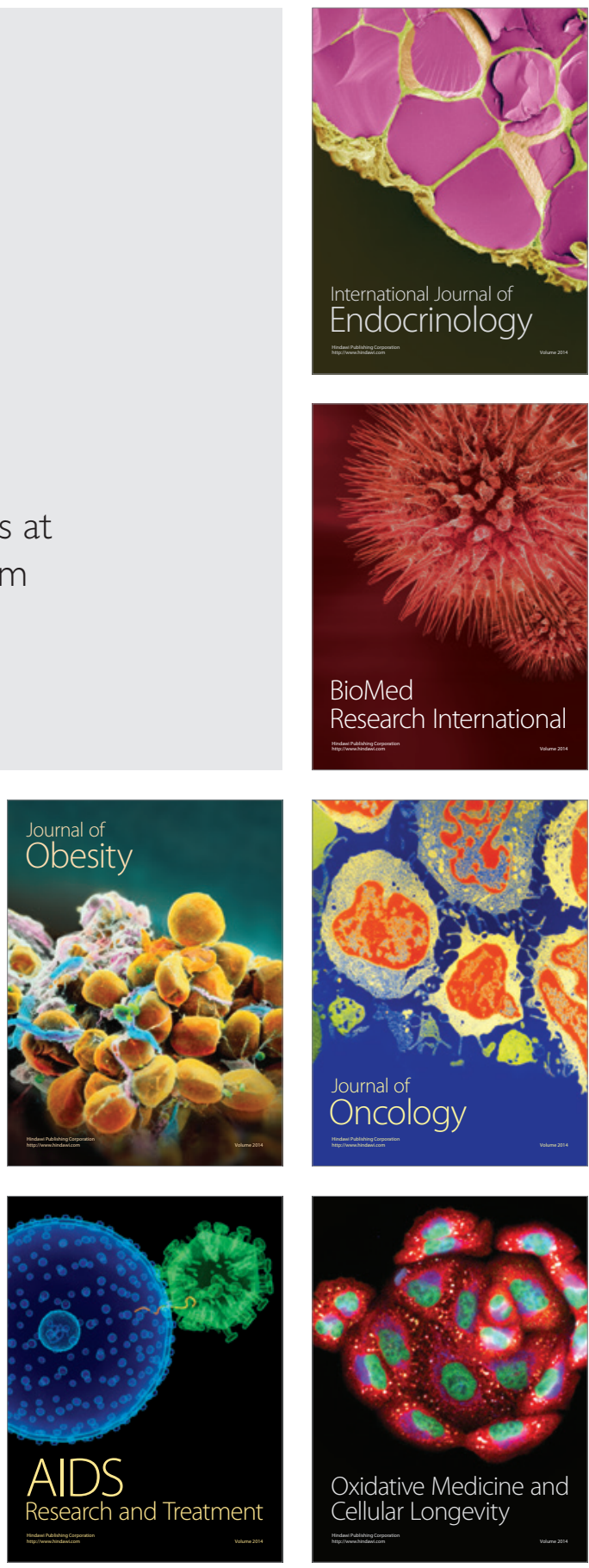